\title{
CORRELACIÓN ENTRE LA ENFERMEDAD RESPIRATORIA AGUDA (ERA) EN MUJERES EMBARAZADAS Y LA CALIDAD DEL AIRE
}

\section{CORRELATION BETWEEN ACUTE RESPIRATORY DISEASE (ARE) IN PREGNANT WOMEN AND THE AIR QUALITY}

\begin{abstract}
William Onatra ${ }^{1}$, Sandra Vargas², Eduardo Páez ${ }^{3}$, Diana Rojas ${ }^{4}$ Angélica López ${ }^{5}$
${ }^{1}$ MD. M.Sc. Biología Reproductiva. Ginecólogo-Endocrinólogo. Profesor Titular. Docente Investigador de Ginecología y Obstetricia. Facultad de Medicina, Universidad de Ciencias Aplicadas y Ambientales U.D.C.A. Dirección para correspondencia: Calle 222 No 55-37. wonatra@gmail.com, ${ }^{2}$ MD. M.Sc Salud Pública. Docente Facultad de Medicina, U.D.C.A, svargas@ udca.edu.co, ${ }^{3}$ MD. M.Sc. Docente de Epidemiología. Facultad de Medicina, U.D.C.A, eduardopaezmd@hotmail.com, ${ }^{4}$ MD. M.Sc Epidemióloga Clínica. Docente de Epidemiología, Facultad de Medicina, U.D.C.A, dianarojasa@gmail.com; ${ }^{5}$ Bióloga. Especialista en Estadística. Departamento de Planeación, U.D.C.A, mariangelica@gmail.com.
\end{abstract}

Rev. U.D.C.A Act. E Div. Cient. 12 (2): 27-37, 2009

\section{RESUMEN}

La contaminación ambiental es uno de los factores que contribuye a exacerbar las enfermedades respiratorias (ERA) y que puede afectar el embarazo. Se tomaron los registros de consulta externa y de urgencias del Hospital de Suba y se tabularon las cinco primeras causas de ERA, correlacionándolas con los promedios mensuales del material particulado PM10, concentración de Gases $\mathrm{NO}, \mathrm{NO}_{2}, \mathrm{NO}_{x}, \mathrm{SO}_{2}$, flujo radiante $(\mathrm{W})$, dirección del viento (DV) y temperatura medio ambiente (TPM). En la evaluación estadística, se utilizaron medidas de tendencia central y el índice de correlación de Pearson. Se tabularon 6.310 casos que ingresaron al Hospital, de los cuales, 1.307 correspondieron a mujeres embarazadas (27,9\%). Las cinco primeras causas de ERA presentaron una frecuencia de asma no específica $(26,1 \%)$, amigdalitis $(24,4 \%)$ gripa $(21,8 \%)$, faringitis (16\%) y asma (11,5\%). Las diferencias promedio de las variables mostraron diferencias entre el primer y el segundo semestre, así: W ( $X=177$ vs 155$)$, DV $(225,7$ vs 199,5$), \mathrm{T}^{\circ} \mathrm{C}(16,8$ vs 15,6$)$ y $\mathrm{PM} 10(94,23$ vs 84,86$)$, y para los gases: $\mathrm{NO}(10,5$ vs 15,2$), \mathrm{NO}_{2}(15,9$ vs 16,4$)$, $\mathrm{NO}_{x}(28,5$ vs 31,8$)$ y SO 2 (2,0 vs 3,2). En su correlación mostró una fuerte asociación entre PM10, $\mathrm{SO}_{2}$ y asma no específica ( $<<0,007$ y 0,0048) y DV y estado gripal $(0,048)$. Se puede afirmar que la contaminación ambiental, se manifiesta en forma cíclica durante el año, afectando la ERA en la mujer embarazada, existiendo una correlación significativa entre las PM10, $\mathrm{SO}_{2}$ y asma no específica y la DV y los estados gripales.

Palabras clave: Enfermedad respiratoria aguda (ERA), embarazo, contaminación del aire.

\section{SUMMARY}

Environmental pollution is one of the factors that contribute to exacerbate respiratory diseases (ERA) and can affect pregnancy. The records of outpatient and emergency entrances of the Hospital of Suba, were studied and tabulated, correlating the five leading causes of ERA with the monthly averages of particulated materials PM10s, fume concentration $\mathrm{NO}, \mathrm{NO}_{2}, \mathrm{NO}_{x}$, $\mathrm{SO}_{2}$, radiant flux $(\mathrm{W})$, wind direction (DV), environmental temperature (TPM). In the evaluation, statistical measures of central tendency and the Pearson index were used. 6310 cases entering the Hospital were tabulated, corresponding 1,307 (27.9\%) to pregnant women. The five leading causes of ERA presented a frequency of non 
specified asthma (26.1\%), tonsillitis (24.4\%) flu (21.8\%), pharyngitis (16\%) and asthma (11.5\%). Variation average of the variables showed differences between the first and the second term: $\mathrm{W}(\mathrm{X}=\mathrm{X} 1.77 \mathrm{vs}=1.55)$, DV (225.7 vs. 199.5), $\mathrm{T}^{\circ} \mathrm{C}$ (16.8 vs. 15.6$)$ and $\mathrm{PM} 10$ (94.23 vs. 84.86 ); for the fumes $\mathrm{NO}(10.5 \mathrm{vs} .15 .2), \mathrm{NO}_{2}$ (15.9vs16.4), $\mathrm{NO}_{\mathrm{x}}$ (28.5 vs. 31.8) and $\mathrm{SO}_{2}$ (2.0 vs 3.2). The correlation showed a strong association between PM10, $\mathrm{SO}_{2}$ and non specific asthma ( $<<0.007$ and 0.0048$)$ and DV and flu state (0.048) It was concluded that the environmental pollution is present in a cyclical manner during the year affecting the ERA in pregnant women, existing a significant correlation between PM10, $\mathrm{SO}_{2}$ and non specific asthma and, DV and statements flu.

Key words: Acute respiratory disease (ARD), pregnancy, air pollution.

\section{INTRODUCCIÓN}

Existe evidencia que la contaminación afecta la salud humana (Künzli et al. 2000; Pope et al. 2002). En el caso específico, los problemas generados por el rápido y desordenado proceso de urbanización de la ciudad de Bogotá D.C., las condiciones sanitarias, la marginalidad, la inequidad, los factores demográficos, ambientales, socio-económicos y los estilos de vida nocivos son determinantes en el deterioro de la salud respiratoria (SDBS, 2006).

La mayoría de estudios se han centrado enlos efectos de la contaminación del aire sobre la mortalidad y la morbilidad del aparato respiratorio (Schatz, 1999). Existen grupos poblacionales más susceptibles que merecen mayor atención, como los ancianos (Saldiva et al. 1995), los niños (Dockery \& Pope, 1994; Heinrich et al. 1999; Schwartz et al. 1994) y las embarazadas (Glinianaia et al. 2004; Maisonet et al. 2004), quienes tienen riesgo de sufrir Enfermedad Respiratoria Aguda (ERA), que se exacerba con los cambios estacionales y de temperatura.

La Organización Mundial de la Salud (OMS) calcula que unos 1.500 millones de individuos están expuestos a polución del aire, causante del $20-30 \%$ de las enfermedades respiratorias; de éstas, 500.000 muertes anuales se deben a contaminantes externos, como partículas (PM10) y dióxido de azufre $\left(\mathrm{SO}_{2}\right)$ y dos millones, a las altas concentraciones de partículas en interiores, atribuyéndose en el embarazo el 4-8\%, de las muertes prematuras (CEPIS-OPS, 2002; McMichael et al. 2003).

La contaminación por partículas sólidas en suspensión (PSS) afecta a la población mundial de forma más permanente que cualquier otro contaminante (CEPIS, 2000). Algunos efectos específicos, como la enfermedad cardiovascular, debida al monóxido de carbono (CO), se puede atribuir directamente a la exposición a contaminantes distintos a las PSS. También son contaminantes dañinos los compuestos inorgánicos gaseosos, como el $\left(\mathrm{SO}_{2}\right)$, el monóxido de carbono (CO), el dióxido de nitrógeno $\left(\mathrm{NO}_{2}\right)$ y algunos hidrocarburos $(\mathrm{HC})$, como el benceno y el butadieno; por ejemplo, el $\mathrm{SO}_{2}$, se puede oxidar y disolver en el agua, formando nieblas de ácido sulfúrico. Las reacciones entre los óxidos de nitrógeno $\left(\mathrm{NO}_{2}\right)$ y los $\mathrm{HC}$ en presencia de la luz solar producen ozono $\left(\mathrm{O}_{3}\right)$, principal componente nocivo del los vapores fotoquímicos (CEPIS-OPS, 2002).

Durante el embarazo, por efecto de las hormonas placentarias, existe un estado de aumento de la respuesta inmune que hace a la mujer susceptible a las infecciones virales, bacterianas y, por ende, a los cambios ambientales, afectando las vías respiratorias (D'Amato $\varepsilon$ Cecchi, 2008). Por otro lado, anatómicamente, existe una disminución de la caja toráxica con aumento del diafragma produciendo un estado de hipoventilación. a medida que progresa el embarazo. La madre responde con una reducción de la tensión de $\mathrm{CO}_{2}\left(\mathrm{PCO}_{2}\right)$ o con hipocapnia con la excreción renal de bicarbonato, determinando una alcalosis respiratoria (Burrow $\mathcal{E}$ Ferris, 1995). Además de los cambios fisiológicos descritos, la embarazada puede desarrollar enfermedades respiratorias agudas, que pueden ser causa de morbi-mortalidad materna y fetal (Dolk et al. 2000; Cabello et al. 2003). Muchos de los cuadros agudos, se presentan con signos y con síntomas que recuerdan los cambios propios de la gestación y, por lo tanto, pueden pasar inadvertidos. Entre estas enfermedades, se destaca el asma bronquial (Schatz, 1999), la neumonía (Berkowits \& Sala, 1990; Rodríguez $\mathcal{E}$ Niederman, 1992), el tromboembolismo pulmonar (TEP) y la trombosis venosa profunda (TVP) (Danilenko-Dixon et al. 2001).

La mayoría de investigaciones están orientadas en el impacto de la contaminación ambiental sobre el 
desarrollo fetal. La relación durante el embarazo ha mostrado relaciones significativas del tipo exposiciónrespuesta, entre la exposición materna a $\mathrm{SO}_{2}$ y la amenaza de parto pretérmino, bajo peso al nacer, observaciones detectadas en estudios conducidos en China, por Wang et al. (1997) y en la República Checa (Bobak, 2000). Por otra parte, la exposición a PM10 ha sido relacionada al retardo del crecimiento intrauterino (RCIU), en un estudio realizado en el norte de Bohemia, sugiriendo un efecto dañino, de carácter permanente, dado que el bajo peso al nacer y RCIU fueron asociados con la salud respiratoria, en etapas posteriores de la vida (Delmeek et al. 1999; Medeiros \& Gouvenia, 2005). RCIU puede llevar a una susceptibilidad exagerada a la exposición a la contaminación del aire y a otros factores ambientales (Gold et al. 1999). Fue de interés de los autores describir si existía una relación entre las mediciones de gases en la estación Corpas de monitoreo, localizada en Suba y la frecuencia de enfermedad pulmonar en mujeres embarazadas quienes consultaron al Hospital de esta localidad, durante el 2005.

\section{MATERIALES Y MÉTODOS}

Es un estudio descriptivo de corte transversal de correlación entre los registros de gases en la estación Corpas de la Localidad de Suba y la frecuencia de las cinco primeras causas de enfermedad respiratoria en mujeres embarazadas, quienes acudieron al Hospital de Suba. El Hospital de Suba, se encuentra a, aproximadamente, $0,4 \mathrm{~km}$ de la estación de monitoreo Corpas y la cobertura para embarazadas es del $72,3 \%$.

Medidores: Existen 14 medidores de monitoreo de la calidad del aire en la ciudad de Bogotá, D.C. Por localización en el área de influencia de la Universidad de Ciencias Aplicadas y Ambientales, se eligió la estación 11 Corpas, por ser ésta la tercera zona del distrito que ofrece mayor contaminación, después de Puente Aranda y de Kennedy. Esta estación esta localizada al noroccidente del Distrito, a una distancia de $13 \mathrm{~km}$ del centro de la ciudad y una extensión de 4.372.23ha, con alto tráfico vehicular y de uso comercial y residencial. Estas estaciones poseen equipos automatizados que cumplen con las regulaciones de US-EPA, analizadores de gases, muestrares que determinan el material particulado (PM10) y sensores metereológicos. En esta estación, se realiza monitoreo diario, semanal y mensual de flujo radiante (W) en $\mathrm{m} / \mathrm{seg}$, la dirección del viento (DV) en grados (Rosa de los vientos), la temperatura del medio ambiente (TMA, $\left.{ }^{\circ} \mathrm{C}\right)$, material particulado PM10 $\left(\mu \mathrm{g} / \mathrm{m}^{3}\right)$, considerándose normal $80 \mu \mathrm{g} /$ $\mathrm{m}^{3}$ anual y, en gases, el $\mathrm{NO}$, el $\mathrm{NO}_{2}, 53$ partículas por billón (PPB), monóxido de nitrógeno oxidado $\left(\mathrm{NO}_{\mathrm{x}}\right)$ y el $\mathrm{SO}_{2} 31 \mathrm{PPB}$ o $80 \mu \mathrm{g} / \mathrm{m}^{3}$ (SDBS, 2006).

Registros hospitalarios: En el Hospital de Suba, se llevó a cabo el registro de la Enfermedad Respiratoria Aguda (ERA), de acuerdo al Manual de Registro de Enfermedades (MRE). Se tabularon, del registro de la base de datos que posee el hospital, las cinco primeras causas más frecuentes de ERA que ingresaron a la Consulta externa y Urgencia de mujeres embarazadas, del primero de enero al 31 de diciembre de 2005.

Comité de ética: El proyecto fue avalado por el Comité de Bioética de la Facultad de Medicina de la U.D.C.A.

Estadísticas: La información fue procesada mediante el programa estadístico de libre acceso "Statistical Package for the Social Sciences (SPSS) 2006". Para las pruebas estadísticas del análisis de la información, se utilizaron medidas de tendencia central; para el cruce de variables, se elaboraron tablas de contingencia y, a partir de éstas, se determinó el índice de correlación, mediante la prueba de chi-cuadrado. Se pretendió un análisis multivariado, aplicando modelos de correlación entre ambos grupos de datos (enfermedades respiratorias y niveles de contaminación) y evaluando cuán fuertes fueron los índices de correlación para las variables, aplicando la prueba de Pearson.

\section{RESULTADOS Y DISCUSIÓN}

De los 6.310 casos que ingresaron a la consulta externa y a urgencias del Hospital de Suba, 3.342 (52,96\%) correspondieron a niños menores de cinco años, 1.661 $(26,32)$ a la población del adulto mayor de 60 años y, a embarazadas, con promedio de edad de 24,5 años, $1.307(20,71 \%)$ casos.

Como se registra en la tabla 1 , la frecuencia de las cinco primeras causas de ERA durante el embarazo por meses muestra que el asma no específica ocupa el $26,1 \%$, la amigdalitis, el 24,4\%, la gripa, 21,8\%, la faringitis $16 \%$ y el asma, 11,5\%. El comportamiento de estas entidades, en el transcurso del año, indica unas curvas bimodales. Se evidencia un incremento del estado gripal en mayo y octubre, que coincide con los periodos de aumento 
de las lluvias para ese año; con la faringitis, se detectó un aumento en enero y con mayor frecuencia en abril, mayo y junio (periodo de lluvia) y dos elevaciones, uno en enero y otro en septiembre (periodo seco). La amigdalitis aparece con mayor frecuencia en enero y en febrero, con un pico en junio y septiembre, que concurre con el tiempo seco; el asma no específica, se manifiesta en enero y febrero (tiempo seco), con un pico en mayo y otro entre septiembre y octubre (final del tiempo seco y aumento de las lluvias); el asma, se presenta durante los periodos secos y su frecuencia se incrementa en el segundo semestre del año: junio y agosto-septiembre y octubre. Es claro que la ERA durante el embarazo manifiesta un aumento de su frecuencia durante los periodos secos y la interfase durante el periodo de lluvias.
Se detectó que existe un mayor número de consultas en el primer semestre, 397 casos (56,3\%) frente al segundo, 307 (43,6\%), no encontrándose diferencias estadística en ERA: estados gripales $(p<0,468)$, faringitis $(p<0,106)$, amigdalitis $(p<0,146)$, asma no específica $(p<0,128)$ y asma $(p<0,321)$. Al hacer evaluación por cuatrimestre, se estipuló que existen diferencias significativas de asma entre enero y mayo $(p<0,003)$ y en asma no específica, en el periodo mayo y septiembre $(p<0,049)$.

Una de las características propias del embarazo es la inmuno-supresión por efectos endocrinos de las hormonas placentarias. Los cambios medio-ambientales favorecen un desequilibrio en el árbol respiratorio, lo que explicaría los efectos encontrados, como los ocasionados por las lluvias, que aumentarían la humedad en abril -

Tabla 1. Frecuencia de las cinco primeras causas de enfermedad pulmonar de mujeres embarazadas entre 10 y 40 años. Hospital de Suba, 2005.

\begin{tabular}{|c|c|c|c|c|c|c|c|c|c|c|c|c|}
\hline \multirow[t]{2}{*}{$\begin{array}{c}\text { MESES } \\
2005\end{array}$} & \multicolumn{2}{|c|}{ Gripa } & \multicolumn{2}{|c|}{ Faringitis } & \multicolumn{2}{|c|}{ Amigdalitis } & \multicolumn{2}{|c|}{$\begin{array}{l}\text { Asma no } \\
\text { Específica }\end{array}$} & \multicolumn{2}{|c|}{ Asma } & \multicolumn{2}{|c|}{ Total } \\
\hline & $\mathrm{n}$ & $\%$ & $n$ & $\%$ & $\mathrm{n}$ & $\%$ & $\mathrm{n}$ & $\%$ & $n$ & $\%$ & $\mathrm{n}$ & $\%$ \\
\hline 01 Enero * & 11 & 7,14 & 13 & 11,50 & 21 & 12,21 & 19 & 10,33 & 3 & 3,70 & 67 & 9,51 \\
\hline 02 Febrero* & 9 & 5,84 & 7 & 6,19 & 20 & 11,63 & 26 & 14,13 & 8 & 9,87 & 70 & 9,94 \\
\hline 03 Marzo & 16 & 10,39 & 6 & 5,30 & 10 & 5,81 & 18 & 9,78 & 3 & 3,70 & 53 & 7,52 \\
\hline 04 Abril + & 6 & 3,8 & 15 & 13,27 & 17 & 9,88 & 8 & 4,34 & 4 & 4,93 & 50 & 7,10 \\
\hline 05 Mayo + & 21 & 13,64 & 13 & 11,50 & 9 & 5,23 & 28 & 15,22 & 8 & 9,87 & 79 & 11,22 \\
\hline 06 Junio & 15 & 9,74 & 14 & 12,38 & 24 & 13,95 & 14 & 7,60 & 11 & 13,58 & 78 & 11,08 \\
\hline 07 Julio * & 13 & 8,44 & 3 & 2,65 & 4 & 2,32 & 8 & 4,34 & 7 & 8,64 & 35 & 4,97 \\
\hline 08 Agosto* & 9 & 5,84 & 8 & 7,07 & 9 & 5,23 & 11 & 5,97 & 10 & 12,34 & 47 & 6,67 \\
\hline 09 Sept. & 9 & 5,84 & 13 & 11,50 & 20 & 11,63 & 20 & 10,87 & 9 & 11,11 & 71 & 10,08 \\
\hline 10 Octubre+ & 24 & 15,58 & 6 & 5,30 & 15 & 8,72 & 21 & 11,41 & 10 & 12,34 & 76 & 10,79 \\
\hline 11 Novi. + & 13 & 8,44 & 8 & 7,07 & 12 & 6,97 & 10 & 5,43 & 6 & 7,40 & 49 & 6,96 \\
\hline 12 Diciembre & 8 & 5,19 & 7 & 6,19 & 11 & 6,39 & 1 & 0,54 & 2 & 2,46 & 29 & 4,11 \\
\hline Total & 154 & 21,87 & 113 & 16,05 & 172 & 24,43 & 184 & 26,13 & 81 & 11,5 & 704 & 100 \\
\hline$p \times c / 6 m$ & & & & & & & & & & & & \\
\hline $\begin{array}{l}p \times \text { cuatri- } \\
\text { mestre }\end{array}$ & & & & & & & & & & & & \\
\hline
\end{tabular}

- Periodos secos + Periodo de lluvias 
mayo y octubre - noviembre, alternando con periodos secos de enero - febrero y julio - agosto, cambios también hallados y descritos por otros autores (Lam, 2007; Laaidi et al. 2006). Se evidencia cómo el flujo radiante es mayor en el primer semestre y disminuye en el segundo, que coincide también con un tendencia en los cambios de temperatura, en promedio de $16^{\circ} \mathrm{C}$, en los primeros meses y $15^{\circ} \mathrm{C}$, en el segundo semestre. La enfermedad respiratoria, en estos dos periodos, muestra un aumento de la amigdalitis y del asma no específica en el primer semestre frente al segundo. Si bien no existe una diferencia significativa de correlación es evidente que hay un comportamiento diferente en estos dos periodos, como anotan otras investigaciones (Sprague E Hagstrom, 1969).

Las variables metereológicas y la concentración de gases registrada en la Red de Monitoreo de la Estación Corpas, se consignaron en la gráfica 1 . En general, las diferentes partículas monitorizadas muestran que los gases, como las PM10, permanecen estables a lo largo del año, con una elevación moderada en noviembre y en diciembre; lo mismo ocurrió con la temperatura, que varió de $1^{\circ} \mathrm{C}$, entre el primer y segundo semestre. La dirección del viento cambia a lo largo de año, con un incremento en abril y en agosto. Al hacer una evaluación entre los dos semestres o entre cuatrimestres, no se hallaron diferencias estadísticas: $\mathrm{NO}(\mathrm{p}<0,150), \mathrm{NO}_{2}(\mathrm{p}<0,284)$, $\mathrm{NO}_{x}(\mathrm{p}<0,261), \mathrm{SO}_{2}(\mathrm{p}<0,101), \mathrm{PM} 10(\mathrm{p}<0,211), \mathrm{W}$ $(\mathrm{p}<0,248)$ y temperatura $(\mathrm{p}<0,090)$.

Las PM10 mostraron un comportamiento bimodal, con un aumento de enero a abril, un promedio bajo entre mayo y octubre y una elevación, nuevamente, en noviembre y en diciembre. Estas partículas, se incrementaron durante la época seca, febrero y marzo, con una tendencia al aumento de la amigdalitis (12\%) y del asma no especifica (13\%). Cuando los niveles están más bajos en junio, julio y agosto (tiempo seco), se presenta un aumento de faringitis (12\%), de amigdalitis (14\%) y de asma (13\%). Al iniciarse un aumento de las PM10 en noviembre y en diciembre (periodo de lluvias), se evidencia una disminución global de la ERA (6-7\%). Las PM10 registran unos valores promedio de enero a abril $(X=101,88)$, de mayo a agosto $(X=79,89)$, con diferencia significativa en este periodo $(p<0,018)$ y entre septiembre y diciembre $(X=86,87)$, sin diferencia significativa $(p<0,234)$. El índice de correlación de
Pearson muestra que las PM10 señalan una fuerte asociación con el asma no específica $(p<0.007)$ (Gráfica 2); otros estudios muestran una restricción de crecimiento intrauterino (Suh et al. 2009).

El diámetro de las partículas presentes en el aire puede variar entre una milésima de micra y 500. Desde el punto de vista del riesgo sobre la salud humana son de mayor interés las partículas, cuyo tamaño no excede las diez micras (PM10), debido a que pueden ingresar al tracto respiratorio y producir daños en los tejidos y en los órganos que lo conforman. La fracción más fina de las PM10 es emitida por vehículos y, especialmente, por el transporte público. Se considera que uno de los factores de mayor gravedad corresponde a la introducción del diesel, de muy mala calidad, como solución a los precios de la gasolina y el cual posee un alto contenido de azufre, 4.500ppm; se espera bajarlo a 1.200ppm, todavía muy alto al ser comparado con la mayoría de los países vecinos donde es de 50-300ppm. Se ha aceptado que las concentraciones máximas anuales de PM10 son de $55 \mu \mathrm{g} / \mathrm{m}^{3}$ (Slama et al. 2008) (Resolución 1208 del 2003 del DAMA); en el D.C., localidades como Kennedy, Puente Aranda, Fontibón y Suba superan los límites permitidos.

El promedio de los gases, en general, es estable a lo largo del año y resalta un aumento en el segundo semestre, especialmente, durante noviembre y diciembre (periodo lluvioso). El comparativo por semestre muestra el NO $(10,5 / 15,2)(p<0,150)$, el NO $(15,9 / 16,4)(p<0,284)$, el

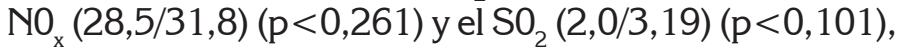
sin diferencias significativas, entre los semestres. Diversos estudios muestran que estos gases elevan los índices de mortalidad perinatal, restricción de crecimiento intrauterino y mortalidad infantil (Woodruff et al. 2009).

Al relacionar el comportamiento de los gases, la ERA registra un aumento de $\mathrm{SO}_{2}$ y $\mathrm{NO}_{x}$ en enero y febrero, donde se manifiesta un incremento de la faringitis (11\%), de la amigdalitis (12\%) y del asma no específica (10\%); en noviembre y diciembre disminuye la ERA, en un $7 \%$. Los gases permanecen estables de mayo a octubre, con un incremento de la frecuencia de faringitis (11\%), de amigdalitis (12\%) y de asma no específica)13\%). El análisis estadístico no evidenció diferencia significativa de los valores de gases monitorizados y ERA en los estados gripales $(p<0,654)$, faringitis $(p<0,974)$, 

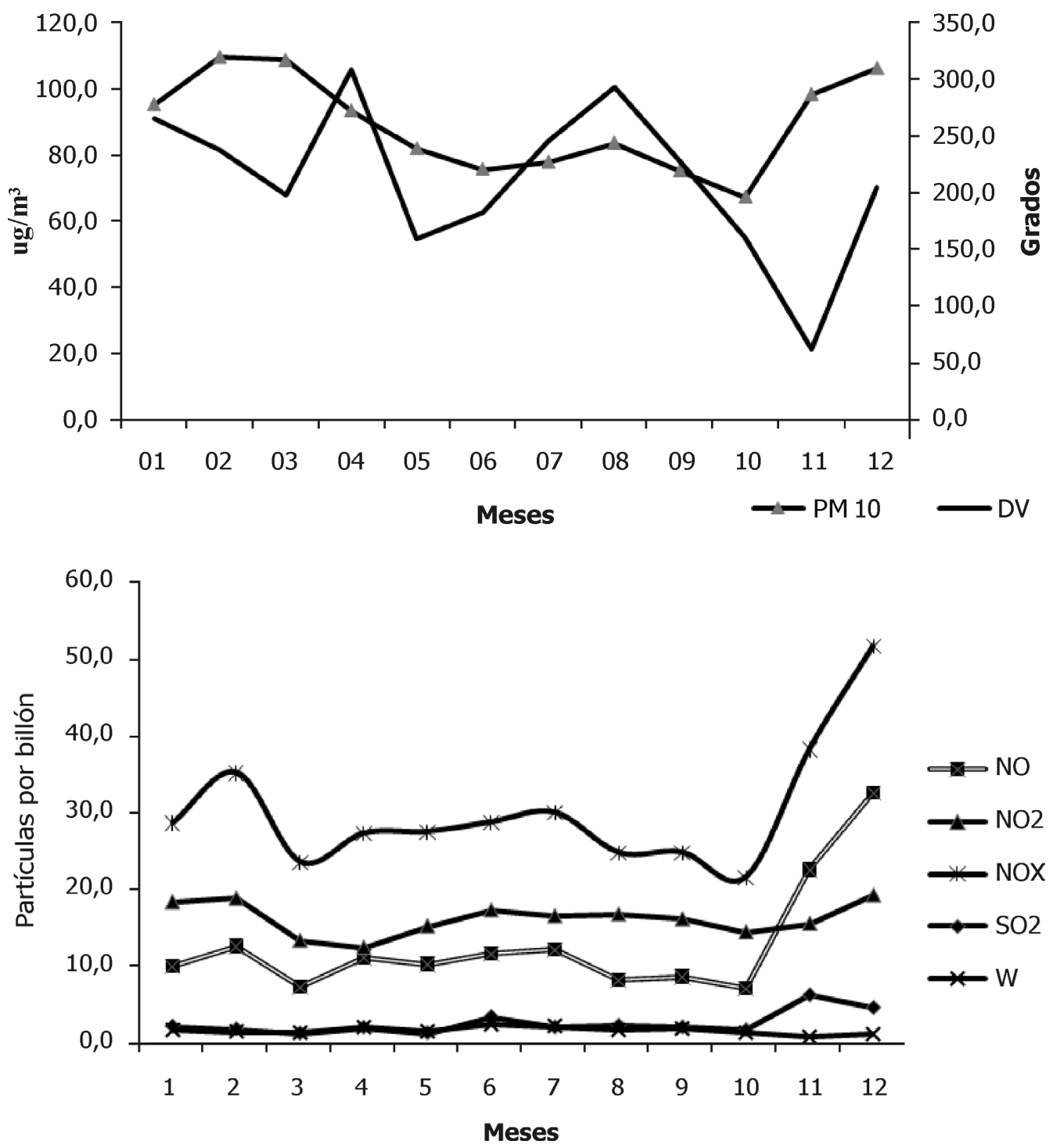

PM 10= material particulado; TMP=Temperatura medio ambiente; $D V=$ Dirección del viento $\mathrm{NO}=$ Monóxido de Nitrógeno; $\mathrm{NO}_{2}=$ Dióxido de Nitrógeno; $\mathrm{NO}_{\mathrm{x}}=$ Monóxido de Nitrógeno oxidado; $\mathrm{SO}_{2}=$ Dióxido de Azufre; $W=$ Flujo radiante

Gráfica 1. Comportamiento de variables metereológicas y de gases en la Estación de Monitorio Corpas de la localidad de Suba, durante el 2005, en Bogotá D.C.

amigdalitis ( $<<0,6845)$, asma no específica $(p<0,249)$ y asma $(p<0,319)$. La correlación de Pearson revela una diferencia significativa con asma no específica con un $\mathrm{R}=0,588$ y una $\mathrm{p}<0,044$ (Gráfica 3).

Con relación a los gases, se sabe que el dióxido de azufre $\left(\mathrm{SO}_{2}\right)$ es incoloro, no inflamable, soluble en agua, puede reaccionar químicamente con otros compuestos en la generación de lluvia ácida y su aspiración continua puede producir problemas respiratorios (Bascom et al. 1996). Se origina, principalmente, durante la combustión de fósiles, como el carbón, la gasolina o el diesel, usados en vehículos y en algunas fábricas.

En este estudio, se confirmó la fuerte asociación entre el $\mathrm{SO}_{2}$ y el asma no específica, con una diferencia 

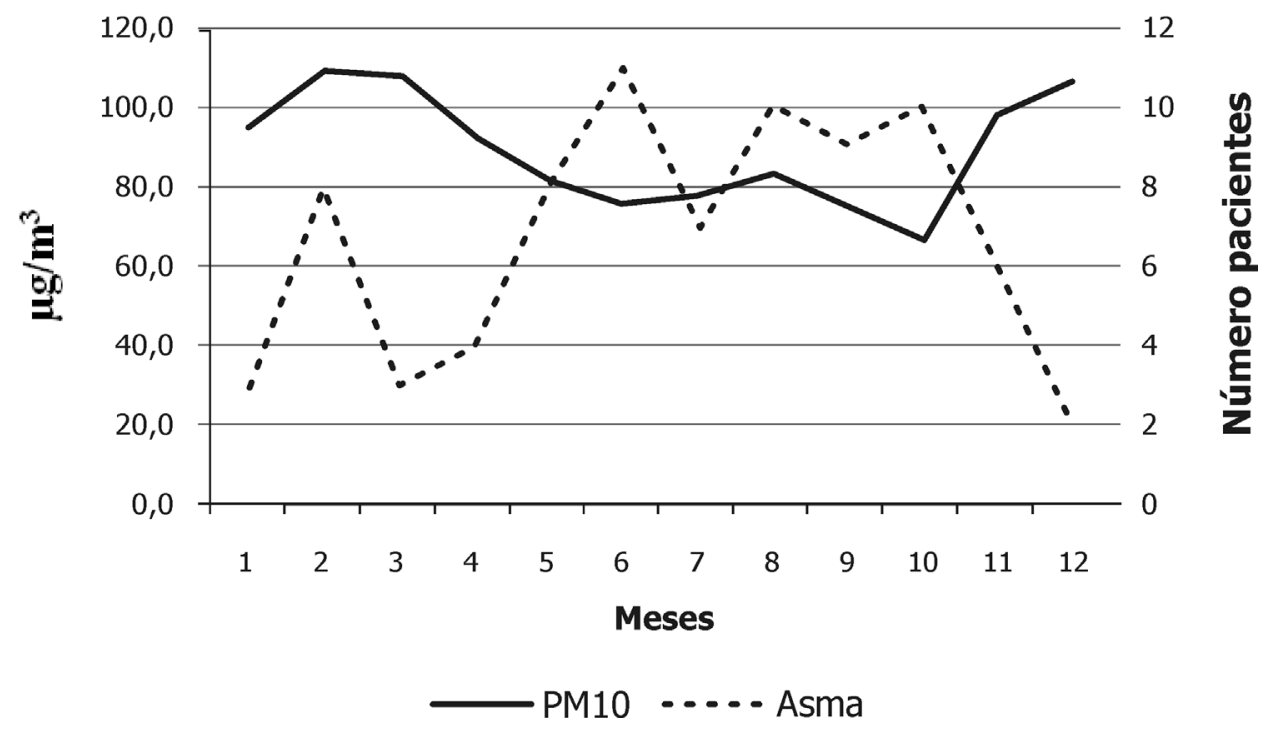

Gráfica 2. Relación entre el material particulado (PM10) y mujeres embarazadas afectadas por asma.
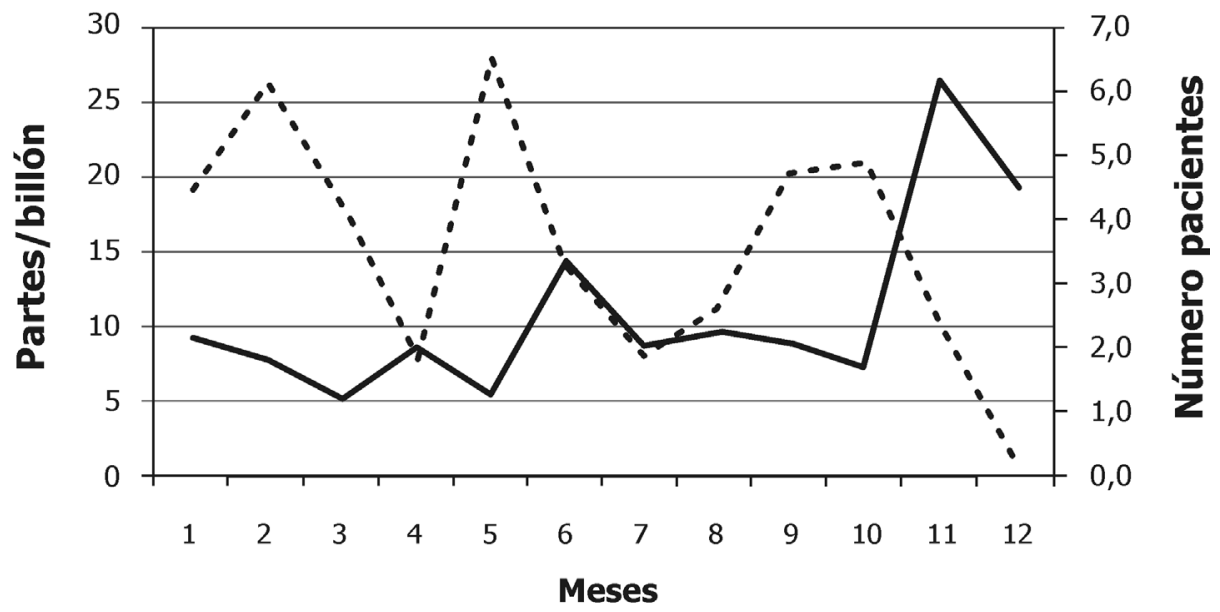

-... Asma no específica - SO2

Gráfica 3. Polución por $\mathrm{SO}_{2}$ y mujeres embarazadas afectadas por asma no específica.

significativa ( $p<0,44)$. Bobak \& León (1999), estudiando la mortalidad infantil neonatal de origen respiratorio, en una población Checa, de acuerdo a los registros de mortalidad y después de hacer los ajustes de covariables biológicos y sociales, encontraron que el RR de la mortalidad de origen respiratorio para $\mathrm{SO}_{2}, 50 \mu \mathrm{g} / \mathrm{m}^{3}$, era $\mathrm{RR}=1,95(95 \% \mathrm{CI}$, $1,09-3,50)$ y para PTS (partículas suspendidas totales), de $\mathrm{RR}=1,74$ (95\% CI, 1,01-2,98). Por otra parte, González et al. (2008) afirman que los países tropicales por sus altas temperaturas y humedad serían un factor importante en las enfermedades respiratorias.

Los gases analizados, el $\mathrm{SO}_{2}$, el $\mathrm{NO}$, el $\mathrm{NO}_{x}$ y el $\mathrm{NO}_{2}$ muestran, en íntesis, una emisión estable durante todo el año, con un aumento de sus niveles a partir de octubre, que coincide con un incremento de la 
ERA. Estadísticamente, se evidenció una diferencia significativa con los estados gripales $(p<0,005)$ y, a pesar de no haber diferencias significativas con la amigdalitis y la faringitis, la elevación de los gases tiene relación con la enfermedad respiratoria. Por otro lado, el asma tiene un aumento de la frecuencia en el segundo semestre del año que coincide con una disminución de la temperatura y de los vientos, favoreciendo los estados alérgicos (HowdenChapman et al. 2008). Se confirma la fuerte asociación significativa entre la DV y los estados gripales $(p<0,048)$. El comportamiento de los estados virales, como la gripa, se exacerban en los periodos lluvia y seco o viceversa, como lo demuestra este estudio, donde aumenta su prevalencia con la llegada del tiempo lluvioso (abril-mayo y octubre-noviembre) (Turcios et al. 2006).

Al comparar las cifras globales con otras ciudades de Colombia y las registradas en la estación Corpas, las PM10 están muy por encima de los niveles permitidos. La tabla 2 registra la emisión de contaminantes al aire en kilotoneladas en las ocho ciudades más contaminadas de Colombia, donde Bogotá ocupa el primer lugar (Solarte et al. 2006).

El flujo radiante (w) muestra un aumento en junio, julio y agosto (periodo seco), evidenciando una tendencia en la frecuencia de la enfermedad respiratoria (estados gripales, faringitis, amigdalitis, asma y asma no específica), en julio y agosto. Al promediar por semestre, se aprecia que el flujo es mayor en el primero $(X=1,77)$ frente al segundo $(X=1,55)$; 397 casos $(56,3 \%)$, en el primer semestre y 307 (43,6\%), en el segundo. Al estimar la correlación estadística no hay diferencias significativas $(p<0,248)$.

La DV muestra un aumento en marzo y abril, que coincide con una mayor frecuencia de faringitis (13\%); en julio, agosto y septiembre, se detecta el asma con más frecuencia (12\%), mientras que en el segundo semestre esto se observa con la faringitis (13\%) y el asma (12\%). En general, se aprecia que en los periodos donde existe un aumento en DV, se presenta una disminución de las enfermedades pulmonares, como en marzo - abril y julio - agosto. En su promedio por semestre es mayor en el primero $(X=225,7$ Grados) frente al segundo (X=199,54 Grados), sin diferencias significativas $(\mathrm{p}<0,238)$. Al aplicar la correlación Pearson, se confirma una diferencia significativa con los estados gripales $(R=0,578)$ y una $p<0,048$ (Gráfica 4).

Por otra parte, la dirección del viento registra unos aumentos en marzo y en agosto, que concuerdan con una disminución de las patologías pulmonares. Es sabido que las enfermedades pulmonares se exacerban en los intervalos en los periodos de lluvias y tiempo seco y es posible que los vapores, por efecto de la temperatura, flujo radiante y viento tengan que ver con este fenómeno (D'Amato E Cecchi, 2008). En el presente estudio, se confirma cómo la dirección del viento presenta una

Tabla 2. Emisiones estimadas de contaminantes al aire, en kilotoneladas en Colombia, 2002.

\begin{tabular}{|c|c|c|c|c|c|}
\hline CIUDAD & PTS & PM-10 & $\mathrm{SO}_{2}$ & $\mathrm{NOx}$ & $\mathrm{CO}$ \\
\hline Normal & & 50 & & & \\
\hline Bogotá & 5,94 & 4,41 & 13,76 & 29,66 & 145,20 \\
\hline Corpas 2005 & & 90,0 & 30,8 & 30,8 & \\
\hline Medellín & 4,52 & 3,11 & 8,85 & 16,34 & 94,56 \\
\hline Cali & 6,91 & 4,44 & 12,81 & 17,91 & 84,77 \\
\hline Barranquilla & 1,67 & 1,66 & 1,41 & 17,23 & 31,52 \\
\hline Sogamoso & 4,51 & 2,61 & 8,10 & 5,79 & 21,99 \\
\hline Bucaramanga & 0,56 & 0,55 & 2,64 & 4,97 & 23,70 \\
\hline Cartagena & 0,45 & 0,45 & 0,73 & 4,61 & 22,88 \\
\hline Pereira & 037 & 0,32 & 0.73 & 2,91 & 19,50 \\
\hline TOTAL CIUD. & 24,93 & 17,55 & 49,03 & 99,41 & 444,12 \\
\hline RESTO PAÍS & 24,27 & 17,88 & 56,11 & 134,97 & 667,81 \\
\hline TOTAL NAL. & 49,21 & 35,43 & 105,14 & 234,38 & $1.111,93$ \\
\hline
\end{tabular}




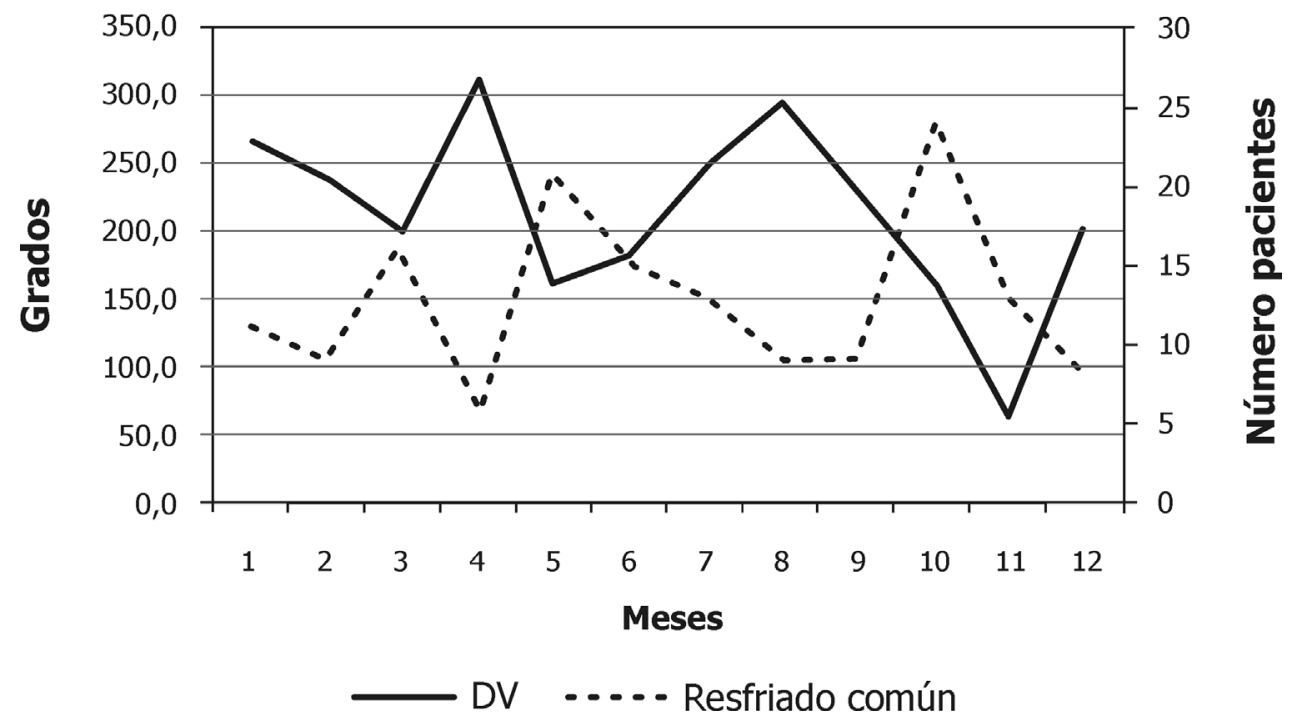

Gráfica 4. Relación entre la dirección del viento (DV) y la afección de mujeres embarazadas por resfriado común.

fuerte asociación, estadísticamente significativa, con el resfriado común $(\mathrm{p}<0,048)$.

La temperatura más fría se registró en mayo $\left(9,7^{\circ} \mathrm{C}\right)$ y su máxima en diciembre $\left(27^{\circ} \mathrm{C}\right)$; se midió un aumento en el primer semestre $\left(X=16,8^{\circ} \mathrm{C}\right)$, con una mayor frecuencia de faringitis (12\%), de amigdalitis (12\%) y de asma no específica $(13 \%)$ y una tendencia a disminuir, $1,2^{\circ} \mathrm{C}$, en el segundo semestre $\left(X=15,6^{\circ} \mathrm{C}\right)$, donde hay una prevalencia mayor de asma ( $12 \%$ ) y de asma no específica (11\%). Estadísticamente no hubo diferencia $(\mathrm{p}<0,090)$.

De acuerdo con estos resultados, se recomienda a las entidades de salud del Distrito tomar medidas de protección respiratoria a las mujeres embarazadas y sus infantes, de la localidad de Suba, especialmente, en la transición entre los periodos secos y lluviosos, vacunación preventiva para la gripe, consulta temprana ante síntomas respiratorios, controlar con filtros los sitios de contaminación de material particulado, dieta rica en verduras, líquidos, habitaciones aireadas y control prenatal desde el primer mes.

AGRADECIMIENTOS: A las directivas y a la Vicerrectoría de Investigaciones de la U.D.C.A, a los directivos y personal administrativo del Hospital de Suba, al personal del Distrito en el área de medio ambiente por su apoyo para llevar a cabo esta investigación. Conflictos de intereses: El manuscrito fue preparado y revisado con la participación de todos los autores, quienes declaramos que no existe ningún conflicto de intereses que ponga en riesgo la validez de los resultados presentados. Financiación: El estudio fue financiado por la Universidad de Ciencias Aplicadas y Ambientales U.D.C.A.

\section{BIBLIOGRAFÍA}

1. BASCOM, R.; BROMBERG, P.; COSTA, D.; BECKER, S.; DEVLIN, R.; KOREN, H.S. 1996. Health effects of outdoor air pollution. Am. J. Respir. Crit. Care Med. 153:477-498.

2. BERKOWITS, K.; LA SALA, A. 1990. Risk factors associated with the increasing prevalence of pneumonia during pregnancy. Am. J. Obstet. Gynecol. 163:981-985.

3. BOBAK, M. 2000. Outdoor air pollution, low birth weight, and prematurity. Environ Health Perspect. 2:173-176.

4. BOBAK, M.; LEÓN, D.A. 1999. The effect of air pollution on infant mortality appears specific for respiratory causes in the postneonatal period. Epidemiology. 10:666-670. 
5. BURROW, G.N.; FERRIS, B.G. 1995. Medical complications during pregnancy. W. Saunders Co. Philadelphia USA: p.327-332.

6. CABELLO, H.A.; MANIEU, D.M.; RUIIZ, M.C. 2003. Enfermedades Respiratorias y Embarazo. Rev. Chil. Enf. Respir. 19:160-165.

7. CEPIS. 2000. Red Panamericana de Muestreo de la Contaminación del Aire (REDPANAIRE). Calidad del Aire: Guías. Plan Regional sobre calidad del aire urbano y salud para el periodo 2000-2009. OPS Colombia - Contaminación Ambiental. Disponible desde Internet en: www.col.ops oms.org/saludambiental/ calidadambiental.asp (con acceso 30/10/08).

8. CEPIS-OPS. 2002. Amaya M.J. Control de la contaminación atmosférica en Bogotá. Octubre 2000. Calidad del aire, perfiles de los países, experiencias de los países, Colombia. Disponible desde Internet en: www.bvsde.ops-oms.org/bvsacep/e/areas.html (con acceso 30/10/08).

9. D `AMATO, G.; CECCHI, L. 2008. Effects of climate change on enviromental factors in respiratory disease. Clin. Exp. Allergy. 38:1264-1274.

10. DANILENKO-DIXON, D.R.; HEIT, J.A.; SILVERSTEIN, M.D.; YAWN, B.P.; PETTERSON, T.M.; LOHSE, C.M. 2001. Risk factors for deep vein thrombosis and pulmonary embolism during pregnancy or post partum: A population-based, case-control study. Am. J. Obstet. Gynecol. 184:104-110.

11. DELMEEK, J.; SELEVAN, S.G.; BENES, I.; SOLANSKY, I.; SRAM, R.J. 1999. Fetal growth and maternal exposure to particulate matter during pregnancy. Environ. Health Perspect. 107:475-480.

12. DOCKERY, D.W.; POPE, C.A. III. 1994. Acute respiratory effects of particulate air pollution. Ann. Rev. Public Health. 15:107-132.

13. DOLK, H.; PATTENDEN, S.; VRIJHEID, M.; THAKRAR. B.; ARMSTRONG, B. 2000. Perinatal and infant mortality and low birth weight among residents near cokeworks in Great Britain. Arch. Environ. Health. 55:26-30.

14. GLINIANAIA, S.V.; RANKIN, J.; BELL, R.; PLESSMULLOLI, T.; HOWEL, D. 2004. Particulate air pollution and fetal health. A systematic review of the epidemiologic evidence. Epidemiology.15:36-45.

15. GOLD, D.R.; BURGE, H.A.; CAREY, V.; MILTON, D.K.; PLATTS-MILLS, T.; WEISS, S.T. 1999. Predictors of repeated wheeze in the first year of life: the relative roles of cockroach, birth weight, acute lower respiratory illness, and maternal smoking. Am. J. Resp. Crit. Care Med 160:227-236.

16. GONZÁLEZ, D.A.; VICTORA, C.G.; GONGALVES, H. 2008. The effects of season at time of birth on asthma and pneumonie and adulthood in a birth cohort in southern Brazil. Cad. Saude Publica. 24:1089-1102.

17. HEINRICH, J.; HOELSCHER, B.; WJST, M.; RITZ, B.; CYRYS, J.; WICHMANN, H. 1999. Respiratory diseases and allergies in two polluted areas in East Germany. Environ. Health Perspect. 107:53-62.

18. HOWDEN-CHAPMAN, P.; PIERSE, N.; NICHOLLS, S.; GILLESPIE-BEENETT, J.; VIG, M.; PHIPPS, R.; BOULIC, M.; FJALLTRON, P.; FREE, S.; CHAPMAN, R.; LLOYD, B.D.; BAKER, M.; CUNNINGHAM, C.; WOODWARD, A.; BULLEN, C.; CRANE, J. 2008. Effects of improved home heating on asthma in community randomised controlled trial. British Med. J. 337:1411.

19. KÜNZLI, N.; KAISER, R.; MEDINA, S.; STUDNICKA, M.; CHANEL, O.; FILLINGER, P. 2000. Publichealth impact of outdoor and traffic-related air pollution: a european assessment. Lancet. 356:795-801.

20. LAAIDI, M.; LAAIDI, K.; BENSANCENOT, J.P. 2006. Temperature related mortality in France, a comparison between different climates from the perspective of global warming. Int. J. Biometeorol. 51:145-153.

21. LAM, L.T. 2007. The association between climatic factors and childhood illnesses presented to hospital emergency among young children. Int. J. Environ. Health Res. 17:1-8.

22. MAISONET, M.; CORREA, A.; MISRA, D.; JAAKKOLA, J.J.K. 2004. A review of the literature on the 
effects of ambient air pollution on fetal growth. Environ. Res. 95:106-115.

23. McMICHAEL, A.J.; CAMPBELL-LENDRUM, D.H.; CORVALÁN, C.F.; EBI, K.L.; GITHEKO, A.; SCHERAGA, J.D.; WOODWARD, A. 2003. Climate change and human health - risks and responses. World Health Organization (WHO). 322p.

24. MEDEIROS, A.; GOUVENIA, N. 2005. Relación entre el bajo peso al nacer y la contaminación del aire en la ciudad de Sao Paulo. Rev Saude Publica 39:965-972.

25. POPE, C.A.; BURNETT, R.T.; THUN, M.J.; CALLE, M.J.; KREWSKI, D.; ITO, K. 2002. Lung cancer, cardiopulmonary mortality, and long-term exposure to fine particulate air pollution. JAMA. 287:1132-1141.

26. RODRÍGUEZ, J.; NIEDERMAN, M. 1992. Pneumonia complicating pregnancy. Clin. Chest. Med. 4: 679-691.

27. SALDIVA, P.H.N.; POPE, C.A. III; SCHWARTZ, J.; DOCKERY, D.W.; LICHTENFELS, A.J.F.; SALGE, J.M. 1995. Air pollution and mortality in elderly people: a time series study in São Paulo, Brazil. Arch. Environ. Health. 50:159-163.

28. SDBS SECRETARÍA DISTRITAL DE SALUD DE BOGOTÁ. 2006. Informe Anual de Calidad del Aire 2005. Red de monitoreo de calidad del aire de Bogotá. Informe mensual marzo 2006. p.1-96. Disponible desde Internet en: www.dama.gov.co (con acceso 13/12/06).

29. SCHATZ, M. 1999. Asthma and pregnancy. Lancet. 353:1202-1224.

30. SCHWARTZ, J.; DOCKERY, D.W.; NEAS, L.M.; WYPIJ, D.; WARE, J.H.; SPENGLER, J.D. 1994. Acute effects of summer air pollution on respiratory symptom reporting in children. Am. J. Respir. Crit. Care Med. 150:1234-1242.

31. SLAMA, R.; DARROW, L.; PARKER, J.; WOODRUFF, T.J.; STRICKLAND, M.; NIEUWENHUIJSEN, M.; GLINIANAIA, S.; HOGGATT, K.J.; KANNAN, S.;
HURLEY, F.; KALINKA, J.; SRÁM, R.; BRAUER, M.; WILHELM, M.; HEINRICH, J.; RITZ, B. 2008. Meeting report atmospheric pollution and human reproduction. Environ. Health Perspect. 116:791798.

32. SOLARTE, I.; HERNÁNDEZ, L.J.; ROJAS, N.Y. 2006. Enfermedad Respiratoria Aguda. Boletín ERA No. 22. Secretaría de Salud. 31 Marzo 2006. 22p. Disponible desde Internet en: www.200.75.49.126/ vsp/boletines/ERA/ERA\%20(PDF)/BOLETIN\%20 ERA\%20No\%2022.pdf. (con acceso 13/12/06).

33. SPRAGUE, H.A.; HAGSTROM, R. 1969. The Nashville air pollution study: mortality multiple regression. Arch. Environ. Health. 18:503-507.

34. SUH, Y.J.; KIM, H.; SEO, J.H.; PARK, H.; KIM, Y.J.; HONG, Y.C.; HA, E.H. 2009. Different effects of PM10 exposure on preterm birth by gestational period estimated from time-dependent survival analyses. Int Arch Occup Environ Health 82:613621.

35. TURCIOS, R.M.; CURNS, A.T.; HOLMAN, R.C.; PANDYA-SMITH, I.; LAMONTE, A.; BRESEE, J.S.; GLASS, R.I. 2006. National respiratory and enteric virus surveillance system collaborating laboratories. Pediatr. Infec. Dis. 25:451-454.

36. WANG, X.; DING, H.; RYAN, L.; XU, X. 1997. Association between air pollution and low birth weight: a community-based study. Environ. Health Perspect. 105:514-520.

37. WOODRUFF, T.J.; PARKER, J.D.; DARROW, L.A.; SLAMA, R.; BELL, M.L.; CHOI, H.; GLINIANAIA, S.; HOGGATT, K.J.; KARR, C.J.; LOBDELL, D.T; WILHELM, M. 2009. Methodological issues in studies of air pollution and reproductive health. Environ. Res. 109:311-320.

Recibido: Diciembre 15 de 2008

Aceptado: Noviembre 7 de 2009 